\title{
Analytical treatment of critical collapse in 2+1 dimensional AdS spacetime: a toy model
}

\author{
Gérard Clément $^{a}{ }^{*}$ and Alessandro Fabbri ${ }^{b} \dagger$ \\ ${ }^{(a)}$ Laboratoire de Physique Théorique LAPTH (CNRS), \\ B.P.110, F-74941 Annecy-le-Vieux cedex, France \\ ${ }^{(b)}$ Dipartimento di Fisica dell'Università di Bologna \\ and INFN sezione di Bologna, \\ Via Irnerio 46, 40126 Bologna, Italy
}

March 23, 2001

\begin{abstract}
We present an exact collapsing solution to $2+1$ gravity with a negative cosmological constant minimally coupled to a massless scalar field, which exhibits physical properties making it a candidate critical solution. We discuss its global causal structure and its symmetries in relation with those of the corresponding continously self-similar solution derived in the $\Lambda=0$ case. Linear perturbations on this background lead to approximate black hole solutions. The critical exponent is found to be $\gamma=2 / 5$.
\end{abstract}

*Email: gclement@lapp.in2p3.fr

${ }^{\dagger}$ Email: fabbria@bo.infn.it 


\section{Introduction}

Since its discovery, the BTZ black hole solution [1] of $2+1$ dimensional AdS gravity has attracted much interest because it represents a simplified context in which to study the classical and quantum properties of black holes. A

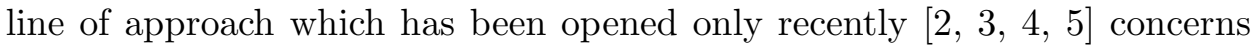
black hole formation through collapse of matter configurations coupled to $2+1$ gravity with a negative cosmological constant. As first discovered in four dimensions by Choptuik [6], collapsing configurations which lie at the threshold of black hole formation exhibit properties, such as universality, power-law scaling of the black hole mass, and continuous or discrete selfsimilarity, which are characteristic of critical phenomena [7]. In the case of a spherically symmetric massless, minimally coupled scalar field, a class of analytical continously self-similar (CSS) solutions was first given by Roberts [8, 9, 10]. These include critical solutions, lying at the threshold between black holes and naked singularities, and characterized by the presence of null central singularities. Linear perturbations of these solutions [11, 12] lead to approximate black hole solutions with a spacelike central singularity.

Numerical simulations of circularly symmetric scalar field collapse in $2+1$ dimensional AdS spacetime were recently performed by Pretorius and Choptuik [2] and Husain and Olivier [3]. Both groups observed critical collapse, which was determined in [2] to be continuously self-similar near $r=0$. In [4], Garfinkle has found a one-parameter family of exact CSS solutions of $2+1$ gravity without cosmological constant, and argued that one of these solutions should give the behaviour of the full critical solution $(\Lambda \neq 0)$ near the singularity.

The purpose of this paper is to present a new CSS solution to the field equations with $\Lambda=0$ which can be extended to a threshold solution of the full $\Lambda \neq 0$ equations. The new $\Lambda=0$ solution is derived in Sect. 3 . It presents a null central singularity and, besides being CSS, possesses four Killing vectors. In Sect. 4 we address the extension of this CSS solution to a quasi-CSS solution of the full $\Lambda<0$ problem, and show that the requirement of maximal symmetry selects a unique extension. This inherits the null central singularity of the $\Lambda=0$ solution, and has the correct AdS boundary at spatial infinity. Finally, we perform in Sect. 5 the linear perturbation analysis in this background, find that it does lead to black hole formation, and determine the critical exponent. 


\section{CSS solutions}

The Einstein equations for cosmological gravity coupled to a massless scalar field in $(2+1)$ dimensions are

$$
G_{\mu \nu}-\Lambda g_{\mu \nu}=\kappa T_{\mu \nu}
$$

with the stress-energy tensor for the scalar field

$$
T_{\mu \nu}=\partial_{\mu} \phi \partial_{\nu} \phi-\frac{1}{2} g_{\mu \nu} \partial^{\lambda} \phi \partial_{\lambda} \phi
$$

The signature of the metric is $(+--)$, and the cosmological constant $\Lambda$ is negative for AdS spacetime, $\Lambda=-l^{-2}$. Static solutions of these equations include the BTZ black hole solutions [1] with a vanishing scalar field $\phi=0$, and singular solutions when a non-trivial scalar field is coupled with the positive sign for the gravitational constant $\kappa[13$.

We shall use for radial collapse the convenient parametrisation of the rotationally symmetric line element in terms of null coordinates $(u, v)$ :

$$
d s^{2}=e^{2 \sigma} d u d v-r^{2} d \theta^{2},
$$

with metric functions $\sigma(u, v)$ and $r(u, v)$. The corresponding Einstein equations and scalar field equation are

$$
\begin{gathered}
r_{, u v}=\frac{\Lambda}{2} r e^{2 \sigma}, \\
2 \sigma_{, u v}=\frac{\Lambda}{2} e^{2 \sigma}-\kappa \phi_{, u} \phi_{, v}, \\
2 \sigma_{, u} r_{, u}-r_{, u u}=\kappa r \phi_{, u}^{2}, \\
2 \sigma_{, v} r_{, v}-r_{, v v}=\kappa r \phi_{, v}^{2}, \\
2 r \phi_{, u v}+r_{, u} \phi_{, v}+r_{, v} \phi_{, u}=0 .
\end{gathered}
$$

From the Einstein equations, the Ricci scalar is

$$
R=-6 \Lambda+4 \kappa e^{-2 \sigma} \phi_{, u} \phi_{, v} .
$$

It follows from (2.9) and (2.5) that the behavior of the solutions near the singularity is governed by the equations (2.4)-(2.8) with vanishing cosmological constant $\Lambda=0$ (see also [5]). Assuming $\Lambda=0$, Garfinkle has found 跑 the following family of exact CSS solutions to these equations

$$
\begin{aligned}
d s^{2} & =-A\left(\frac{(\sqrt{v}+\sqrt{-u})^{4}}{-u v}\right)^{\kappa c^{2}} d u d v-\frac{1}{4}(v+u)^{2} d \theta^{2}, \\
\phi & =-2 c \ln (\sqrt{v}+\sqrt{-u}),
\end{aligned}
$$


depending on an arbitrary constant $c$ and a scale $A>0$. In (2.10), $u$ is retarded time, and $-v$ is advanced time. These solutions are continuously self-similar with homothetic vector $\left(u \partial_{u}+v \partial_{v}\right)$. An equivalent form of these CSS solutions, obtained by making the transformation

$$
-u=(-\bar{u})^{2 q}, \quad v=(\bar{v})^{2 q} \quad\left(1 / 2 q=1-\kappa c^{2}\right)
$$

to the barred null coordinates $(\bar{u}, \bar{v})$, is

$$
\begin{aligned}
d s^{2} & =-\bar{A}\left(\bar{v}^{q}+(-\bar{u})^{q}\right)^{2(2 q-1) / q} d \bar{u} d \bar{v}-\frac{1}{4}\left(\bar{v}^{2 q}-(-\bar{u})^{2 q}\right)^{2} d \theta^{2} \\
\phi & =-2 c \ln \left(\bar{v}^{q}+(-\bar{u})^{q}\right) .
\end{aligned}
$$

The corresponding Ricci scalar is

$$
R=\frac{4 \kappa c^{2}}{A}\left(\bar{v}^{q}+(-\bar{u})^{q}\right)^{2(1-3 q) / q}(-\bar{u})^{q-1}(\bar{v})^{q-1} .
$$

Garfinkle suggested that the line element (2.10) describes critical collapse in the sector $r=-(u+v) / 2 \geq 0$, near the future point singularity $r=0$ (where the Ricci scalar behaves, for $v \propto u$, as $u^{-2}$ ). The corresponding Penrose diagram (Fig. 1) is a triangle bounded by past null infinity $u \rightarrow-\infty$, the other null side $v=0$, and the central regular timelike line $r=0$. For $\kappa c^{2} \geq 1(q<0)$, the Ricci scalar

$$
R \sim(\bar{v})^{q-1} \sim(v)^{(q-1) / 2 q}
$$

is regular near $v=0$, which moreover turns out to be at infinite geodesic distance. To show this, we consider the geodesic equation

$$
\left(e^{2 \sigma} \dot{v}\right)^{\cdot}=-2 r r_{, u} \dot{\theta}^{2}=-2 l^{2} r^{-3} r_{, u}
$$

( $l$ constant) near $v=0, u$ constant, which gives $v \propto(l s)^{4 q}$ for $l \neq 0$, or $s^{2 q}$ for $l=0$, so that in all cases the affine parameter $s \rightarrow \infty$ for $v \rightarrow 0$, and the spacetime is geodesically complete. For $\kappa c^{2}<1(q>0)$, we see from (2.13) that the null line $v=0$ is a curvature singularity if $\kappa c^{2}<1 / 2(q<1)$. If $1 / 2 \leq \kappa c^{2}<1(q \geq 1)$, the surface $v=0$ is regular. However, as discussed by Garfinkle, the metric (2.12) can be extended through this surface only for $q=n$, where $n$ is a positive integer. For $n$ even, the extended spacetime is made of two symmetrical triangles joined along the null side $\bar{v}=0$, and has two coordinate singularities $r=0$, one timelike $(\bar{u}-\bar{v}=0)$ and one spacelike $(\bar{u}+\bar{v}=0)$, but no curvature singularity. For $n$ odd, one of the $r=0$ sides becomes a future spacelike curvature singularity $\left(e^{2 \sigma}=0\right)$, similar to that of 
Brady's supercritical solutions for scalar field collapse in $(3+1)$ dimensions [9], except for the fact that in the present case the singularity is not hidden behind a spacelike apparent horizon (Fig. 2).

Let us point out that, besides the solutions (2.10), the system (2.4)-(2.8) also admits for $\Lambda=0$ another family of CSS solutions

$$
\begin{aligned}
d s^{2} & =A\left(\frac{(\sqrt{v}-\sqrt{-u})^{4}}{-u v}\right)^{\kappa c^{2}} d u d v-\frac{1}{4}(v+u)^{2} d \theta^{2} \\
& =\bar{A}\left(\bar{v}^{q}-(-\bar{u})^{q}\right)^{2(2 q-1) / q} d \bar{u} d \bar{v}-\frac{1}{4}\left(\bar{v}^{2 q}-(-\bar{u})^{2 q}\right)^{2} d \theta^{2}
\end{aligned}
$$

with $\phi=-2 c \ln (\sqrt{v}-\sqrt{-u})$, and we choose $A>0$ and consider the sector $0 \leq v \leq-u$. These solutions have a future spacelike central $(r=0)$ curvature singularity at $(-\bar{u})^{q}=\bar{v}^{q}$ (where the Ricci scalar (2.13) diverges) for all $q<0$ or $q>0$ (implying $q>1 / 2$ ). For $q<0$, the Penrose diagram is a triangle bounded by past null infinities $\bar{u} \rightarrow-\infty$ and $\bar{v}=0$ (which is at infinite geodesic distance). For $q>0$, geodesics terminate at $\bar{v}=0$, unless $q=n$ integer. For $n$ even, the extended spacetime has two central curvature singularities $r=0$, one spacelike and the other timelike. The extended spacetime for $n$ odd is more realistic. In this case the extension from $\bar{v}>0$ to $\bar{v}<0$ amounts to replacing (2.16) with $A>0$ by the original Garfinkle solution (2.10) with $A>0$, the resulting Penrose diagram being that of Fig. 2 .

\section{A new CSS solution for $\Lambda=0$}

Among the one-parameter ( $c$ or $q$ ) family of CSS solutions (2.10), the special solution, corresponding to $\kappa c^{2}=1$,

$$
d s^{2}=A(\sqrt{v}+\sqrt{-u})^{4} \frac{d u}{u} \frac{d v}{v}-\frac{1}{4}(v+u)^{2} d \theta^{2},
$$

is singled out by the fact that the transformation (2.11) breaks down for this value. The transformation appropriate to this case,

$$
-u=2 e^{-U}, \quad v=2 e^{V}=2 e^{U-2 T}
$$

(with $T \geq U$ for $u+v \leq 0$ ) transforms the solution (3.1) to

$$
\begin{aligned}
d s^{2} & =e^{-2 U}\left[-4 A\left(1+e^{U-T}\right)^{4} d U d V-\left(1-e^{2(U-T)}\right)^{2} d \theta^{2}\right] \\
\phi & =U-2 \ln \left(1+e^{U-T}\right)
\end{aligned}
$$


(we use from now on units such that $\kappa=1$, and have dropped an irrelevant additive constant from $\phi$ ).

Starting from this special CSS solution of the Garfinkle class, we now derive, by a limiting process, a new CSS solution which, as we shall see, exhibits a null singularity. We translate $T$ to $T-T_{0}$, and take the late-time limit $T_{0} \rightarrow-\infty$, leading to the new CSS solution (written for $A=-1 / 2$ )

$$
d s^{2}=e^{-2 U}\left(2 d U d V-d \theta^{2}\right), \quad \phi=U,
$$

with a very simple form which is reminiscent of the Hayward critical solution for scalar field collapse in $3+1$ dimensions [12],

$$
d s^{2}=e^{2 \rho}\left(2 d \tau^{2}-2 d \rho^{2}-d \Omega^{2}\right), \quad \phi=\tau .
$$

The transformation

$$
\bar{u}=-e^{-2 U}, \quad \bar{v}=V
$$

leads from (3.4) to the even more simple form of this solution

$$
d s^{2}=d \bar{u} d \bar{v}+\bar{u} d \theta^{2}, \quad \phi=-\frac{1}{2} \ln (-\bar{u}),
$$

which is reminiscent of the other form of the Hayward solution

$$
d s^{2}=2 d \bar{u} d \bar{v}+\bar{u} \bar{v} d \Omega^{2}, \quad \phi=-\frac{1}{2} \ln (-\bar{u} / \bar{v}) .
$$

The solution (3.4) or (3.7) is continuously self-similar, with homothetic vector

$$
K=\partial_{U}=-2 \bar{u} \partial_{\bar{u}} .
$$

It also has a high degree of symmetry, with 4 Killing vectors

$$
\begin{aligned}
& L_{1}=\partial_{U}+2 V \partial_{V}+\theta \partial_{\theta}, \\
& L_{2}=\theta \partial_{V}+U \partial_{\theta}, \\
& L_{3}=\partial_{V}, \\
& L_{4}=\partial_{\theta},
\end{aligned}
$$

generating the solvable Lie algebra

$$
\begin{array}{cl}
{\left[L_{1}, L_{2}\right]=L_{4}-L_{2},} & {\left[L_{2}, L_{3}\right]=0,} \\
{\left[L_{1}, L_{3}\right]=-2 L_{3},} & {\left[L_{2}, L_{4}\right]=-L_{3},} \\
{\left[L_{1}, L_{4}\right]=-L_{4},} & {\left[L_{3}, L_{4}\right]=0 .}
\end{array}
$$


The Ricci scalar (2.9) is identically zero for the solution (3.4), for which the sole nonvanishing Ricci tensor component is $R_{U U}=1$. It follows that this metric is devoid of curvature singularity. However there is an obvious coordinate singularity at $U \rightarrow+\infty$, or $\bar{u}=0$ (where $r=0$ ). To determine the nature of this singularity, we study geodesic motion in the spacetime (3.7). The geodesic equations are integrated by

$$
\dot{\bar{u}}=\pi, \quad \bar{u} \dot{\theta}=l, \quad \pi \dot{\bar{v}}+l \dot{\theta}=\varepsilon,
$$

where $\pi$ and $l$ are the constants of the motion associated with the Killing vectors $L_{3}$ and $L_{4}$, and the sign of $\varepsilon$ depends on that of $d s^{2}$ along the geodesic. The null line $\bar{u}=0$ can be reached only by those geodesics with $\pi \neq 0$. Then, the third equation (3.12) integrates to

$$
\bar{v}=\frac{\varepsilon}{\pi^{2}} \bar{u}-\frac{l}{\pi} \theta+\text { const. }=\frac{\varepsilon}{\pi^{2}} \bar{u}-\frac{l^{2}}{\pi^{2}} \ln (-\bar{u})+\text { const. . }
$$

It follows that nonradial geodesics $(l \neq 0)$ terminate at $\bar{u}=0, \bar{v} \rightarrow+\infty$, while radial geodesics $(l=0)$, which behave as in cylindrical Minkowski space, can be continued through the null line $\bar{u}=0$ to $\bar{u} \rightarrow+\infty$. So in this sense only the endpoint $\bar{v} \rightarrow+\infty$ of the null line $\bar{u}=0$ is singular. However formal analytic continuation of the metric (3.7) from $\bar{u}<0$ to $\bar{u}>0$ involves a change of signature from $(+--)$ to $(+-+)$, leading to the appearance of closed timelike curves. So the null line $\bar{u}=0$ corresponds to a singularity in the causal structure of the spacetime, analogous to the central singularity in the causal structure of the BTZ black holes [1]. The resulting Penrose diagram, reminiscent of that of the Hayward critical solution [12], is a diamond bound by three lines at null infinity $(\bar{v}=-\infty, \bar{u}=-\infty, \bar{v}=+\infty)$ and the null singularity $\bar{u}=0$ (Fig. 3).

\section{Extending the new solution to $\Lambda \neq 0$}

In the preceding section we have found an exact solution for scalar field collapse with $\Lambda=0$, which presents a central null singularity. This property makes it a candidate threshold solution, lying at the boundary between naked singularities and black holes. However black holes exist only for $\Lambda<0$, so the solution (3.7) can only represent the behavior of the true threshold solution near the central singularity, where the cosmological constant can be neglected. This hypothetical $\Lambda<0$ solution cannot be self-similar, essentially because the scale is fixed preferentially by the cosmological constant 
[2]. So what we need is to find some other way to extend (3.7) to a solution of the full system of Einstein equations with $\Lambda<0$.

A first possible approach is to expand this solution in powers of $\Lambda$, with the zeroth order given by the CSS solution (3.7). In the parametrisation (2.3), this zeroth order is (dropping the bars in (3.7) )

$$
r_{0}=(-u)^{1 / 2}, \quad \sigma_{0}=0, \quad \phi_{0}=-\frac{1}{2} \ln |u| .
$$

We look for an approximate solution to first order in $\Lambda$ of the form

$$
r=(-u)^{1 / 2}+\Lambda r_{1}, \quad \sigma_{=} \Lambda \sigma_{1}, \quad \phi=-\frac{1}{2} \ln |u|+\Lambda \phi_{1},
$$

with the boundary condition that the fonctions $r_{1}, \sigma_{1}$ and $\phi_{1}$ vanish on the central singularity $u=0$. Eq (2.4) gives

$$
r_{1}=(-u)^{1 / 2}\left(\frac{1}{3} u v+f(u)\right)
$$

with $f(0)=0$. Then, the linearized Eq. (2.7) gives

$$
2 r_{0}^{1 / 2}\left(r_{0}^{1 / 2} \phi_{1, v}\right)_{, u}=-r_{1, v} \phi_{0, u}=\frac{1}{6}(-u)^{1 / 2},
$$

which is solved by

$$
\phi_{1}=\left(\frac{1}{15} u v+g(u)\right)
$$

The linearized Eq. (2.5)

$$
2 \sigma_{1, u v}=1-\phi_{0, u} \phi_{1, v}=\frac{8}{15}
$$

then gives

$$
\sigma_{1}=\frac{4}{15} u v+h(u)
$$

Finally Eq. (2.5) leads to the relation between the arbitrary functions $f, g$, $h$

$$
u f^{\prime \prime}(u)+f^{\prime}(u)=g^{\prime}(u)+h^{\prime}(u) .
$$

Not only does this first order solution break the continuous self-similarity generated by (3.9), as expected, but it also breaks the isometry group generated by the Killings (3.10) down to $U(1)$ (generated by $L_{4}=\partial_{\theta}$ ), except in the special case $f=g=h=0$, where the Killing subalgebra $\left(L_{1}, L_{4}\right)$ 
remains. This suggests looking for an exact $\Lambda<0$ extension of the $\Lambda=0$ CSS solution of the form

$$
d s^{2}=e^{2 \sigma(x)} d u d v+u \rho^{2}(x) d \theta^{2}, \quad \phi=-\frac{1}{2} \ln |u|+\psi(x),
$$

with $x=u v$. This will automatically preserve to all orders the Killing subalgebra $\left(L_{1}, L_{4}\right)$. Inserting this ansatz into the field equations (2.4)-(2.8) leads to the system

$$
\begin{aligned}
x \rho^{\prime \prime}+\frac{3}{2} \rho^{\prime} & =\frac{\Lambda}{2} \rho e^{2 \sigma}, \\
2\left(x \sigma^{\prime \prime}+\sigma^{\prime}\right)+\psi^{\prime}\left(x \psi^{\prime}-\frac{1}{2}\right) & =\frac{\Lambda}{2} e^{2 \sigma}, \\
x^{2}\left(-\rho^{\prime \prime}+2 \rho^{\prime} \sigma^{\prime}-\rho \psi^{\prime 2}\right)+x\left(-\rho^{\prime}+\rho\left(\sigma^{\prime}+\psi^{\prime}\right)\right) & =0 \\
-\rho^{\prime \prime}+2 \rho^{\prime} \sigma^{\prime}-\rho \psi^{\prime 2} & =0 \\
2 x\left(\rho \psi^{\prime}\right)^{\prime}+\frac{5}{2} \rho \psi^{\prime} & =\frac{1}{2} \rho^{\prime} .
\end{aligned}
$$

$\left({ }^{\prime}=d / d x\right)$. The unique, maximally symmetric extension of the CSS solution (3.7) reducing to (3.7) near $u=0$ is the solution of the system (4.10)-(4.14) with the boundary conditions

$$
\rho(0)=1, \quad \sigma(0)=0, \quad \psi(0)=0 .
$$

The comparison of (4.12) and (4.13) yields

$$
\rho=e^{\sigma+\psi} .
$$

The combination $(4.10)+x(4.13)$ then gives, together with $(4.16)$,

$$
x\left(2 \sigma^{\prime 2}+2 \sigma^{\prime} \psi^{\prime}-\psi^{\prime 2}\right)+\frac{3}{2}\left(\sigma^{\prime}+\psi^{\prime}\right)=\frac{\Lambda}{2} e^{2 \sigma} .
$$

The third independent equation is for instance (4.11):

$$
2\left(x \sigma^{\prime \prime}+\sigma^{\prime}\right)+\psi^{\prime}\left(x \psi^{\prime}-\frac{1}{2}\right)=\frac{\Lambda}{2} e^{2 \sigma} .
$$

Using these last two equations with the boundary conditions (4.15), one can in principle write down series expansions for $\sigma(x)$ and $\psi(x)$. Another simple relation, deriving from (4.13) and (4.16), is

$$
\sigma^{\prime \prime}+\psi^{\prime \prime}-\sigma^{2}+2 \psi^{2}=0 .
$$


We are interested in the behavior of this extended solution in the sector $u<0, v>0$, i.e. $x<0$. In this sector, Eqs. (4.10), (4.14) and (4.11) can be integrated to

$$
\begin{aligned}
(-x)^{3 / 2} \rho^{\prime} & =\frac{\Lambda}{2} \int_{x}^{0}(-x)^{1 / 2} \rho e^{2 \sigma} d x \\
(-x)^{5 / 4} \rho \psi^{\prime} & =\frac{1}{4} \int_{x}^{0}(-x)^{1 / 4} \rho^{\prime} d x \\
-x \sigma^{\prime} & =\frac{1}{2} \int_{x}^{0}\left(\frac{\Lambda}{2} e^{2 \sigma}+\psi^{\prime}\left(\frac{1}{2}-x \psi^{\prime}\right)\right) d x .
\end{aligned}
$$

As long as $\rho>0$, Eq. (4.20) (with $x<0, \Lambda<0$ ) implies $\rho^{\prime}<0$, so that $\rho(x)$ decreases to 1 when $x$ increases to 0 . It then follows from (4.21) that $\psi^{\prime}<0$. Also, (4.21) can be integrated by parts to

$$
x \psi^{\prime}=\frac{1}{4}-\frac{1}{16(-x)^{1 / 4} \rho} \int_{x}^{0}(-x)^{-3 / 4} \rho d x,
$$

showing that $x \psi^{\prime}<1 / 4$. It then follows from (4.22) that $\sigma^{\prime}<0$. So, as $x$ decreases, the functions $\rho$ and $e^{2 \sigma}$ increase and possibly go to infinity for a finite value $x=x_{1}$. If this is the case, the behavior of these functions near $x_{1}$ must be

$$
\begin{aligned}
\rho & =\rho_{1}\left(\frac{1}{\bar{x}}+\frac{1}{4 x_{1}}-\frac{\bar{x} \ln (\bar{x})}{48 x_{1}^{2}}+\ldots\right) \\
e^{2 \sigma} & =\frac{4 x_{1}}{\Lambda \bar{x}^{2}}\left(1+\frac{\bar{x}^{2} \ln (\bar{x})}{48 x_{1}^{2}}+\ldots\right) \\
\psi & =\psi_{1}+\frac{\bar{x}}{4 x_{1}}-\frac{\bar{x}^{2}}{32 x_{1}^{2}} \ln (\bar{x})+\ldots
\end{aligned}
$$

$\left(\bar{x}=x-x_{1}\right)$.

These expectations are borne out by the actual numerical solution of the system

$$
\begin{aligned}
x \rho^{\prime \prime}+\frac{3}{2} \rho^{\prime} & =-\rho e^{2 \sigma}, \\
-\rho^{\prime \prime} \rho+4 \rho \rho^{\prime} \sigma^{\prime} & =\rho^{\prime 2}+\rho^{2} \sigma^{\prime 2},
\end{aligned}
$$

(this last equation comes from (4.13) where $\psi^{\prime}$ is given by derivation of (4.16) ) where we have set $\Lambda=-2$, with the boundary counditions $\rho(0)=1$, $\rho^{\prime}(0)=-2 / 3$ (see eqs. (4.3) and (4.2) ),$\sigma(0)=0$. The plots of the functions 
$\rho(x), \sigma(x)$ and $\psi^{\prime}(x)$ are given in Figs. $(4,5,6$,$) . The value of x_{1}$ is found to be approximately -1.94 (i.e. $\Lambda x_{1}=+3.88$ ).

The coordinate transformation!

$$
u=\Lambda^{-1} e^{-\bar{U}}, \quad v=e^{\bar{V}} \quad(\bar{U}=\bar{T}-\bar{R}, \quad \bar{V}=\bar{T}+\bar{R})
$$

leads to $x=\Lambda^{-1} e^{2 \bar{R}}$ and, on account of (4.9) and (4.16), to the form of the metric

$$
d s^{2}=-\Lambda^{-1} e^{2(\sigma(\bar{R})+\bar{R})}\left(d \bar{U} d \bar{V}-e^{2 \psi(\bar{R})-\bar{V}} d \theta^{2}\right) .
$$

Near the spacelike boundary $\bar{R}=\bar{R}_{1}$ of the spacetime, the collapsing metric and scalar field behave, from (4.24), as

$$
d s^{2} \simeq-\Lambda^{-1}\left(\bar{R}_{1}-\bar{R}\right)^{-2}\left(d \bar{T}^{2}-d \bar{R}^{2}-e^{\bar{T}_{1}-\bar{T}} d \theta^{2}\right), \quad \phi=\phi_{1}+\bar{T} / 2
$$

$\left(\bar{R}-\bar{R} \simeq \bar{x} / 2 x_{1}\right)$. This metric is asymptotically AdS, as may be shown by making the further coordinate transformation,

$$
\bar{R}-\bar{R}_{1}=-2 / X T, \quad \bar{T}-\bar{T}_{1}=2 \ln (T / 2),
$$

leading to

$$
d s^{2} \simeq-\Lambda^{-1}\left(X^{2} d T^{2}-\frac{d X^{2}}{X^{2}}-X^{2} d \theta^{2}\right), \quad \phi=\phi_{1}+\ln (T / 2) .
$$

The next-to-leading terms in the metric containing logarithms, this asymptotic behavior differs from that of BTZ black holes.

It follows from this discussion that the Penrose diagram of the $\Lambda<0$ threshold solution in the sector $v>0, u<0$ is a triangle bounded by the null line $v=0$, the null causal singularity $u=0$, and the spacelike AdS boundary $X \rightarrow \infty$. The null singularity $u=0$ remains naked, i.e. is not hidden behind a trapping horizon, which would correspond to

$$
\partial_{v} r=-(-u)^{3 / 2} \rho^{\prime}(x)=0,
$$

because $\rho^{\prime}<0$ (as discussed above) implies that the only solution of this equation is $u=0$.

For the sake of completeness, let us also discuss the behavior of the solution of the system (4.10)-(4.14) in the sector $x>0$. In this case, one can write down integro-differential equations similar to (4.20)-(4.22), from

\footnotetext{
${ }^{1}$ We have taken care that in (4.9) $u$ has the dimension of a length squared while $v$ is dimensionless.
} 
which one again derives that $\rho^{\prime}<0, \psi^{\prime}<0$ and $\sigma^{\prime}<0$. It follows that the metric function $e^{2 \sigma}$ decreases as $x$ increases, eventually vanishing for a finite value $x=x_{0}$, corresponding to a spacelike curvature singularity (this has been confirmed numerically). The behavior of the solution near this singularity is found to be

$$
\psi \simeq \gamma \ln \left(x_{0}-x\right), \quad \sigma \simeq \frac{\gamma^{2}}{2} \ln \left(x_{0}-x\right), \quad \rho \propto\left(x_{0}-x\right) \quad(\gamma=\sqrt{3}-1),
$$

and the coordinate transformation $u=e^{U}, v=e^{V}\left(x=e^{2 T}\right)$ leads to the form of the metric near the singularity

$$
d s^{2} \simeq\left(T_{0}-T\right)^{\gamma^{2}}\left(d T^{2}-d R^{2}\right)+e^{R_{0}-R}\left(T_{0}-T\right)^{2} d \theta^{2} .
$$

\section{Perturbations}

To check whether the quasi-CSS solution (4.9) of the full $\Lambda \neq 0$ problem determined in the preceding section is indeed a threshold solution, we now study linear perturbations of this solution. Our treatment will follow the analysis of perturbations of critical solutions in the case of scalar field collapse in 3+1 dimensions [11, 12].

The relevant time parameter in critical collapse being the retarded time $U=-(1 / 2) \ln (-u)$ (the "scaling variable" of [11), we expand these perturbations in modes proportional to $e^{k U}=(-u)^{-k / 2}$, with $k$ a complex constant. We recall that only the modes with $R e k>0$ grow as $U \rightarrow+\infty$ $(u \rightarrow-0)$ and lead to black hole formation, whereas those with $\operatorname{Re} k<0$ decay and are irrelevant. The other relevant variable is the "spatial" coordinate $x=u v$, and the perturbations are decomposed as

$$
\begin{aligned}
r & =(-u)^{1 / 2}\left(\rho(x)+(-u)^{-k / 2} \tilde{r}(x)\right), \\
\phi & =-\frac{1}{2} \ln |u|+\psi(x)+(-u)^{-k / 2} \tilde{\phi}(x), \\
\sigma & =\sigma(x)+(-u)^{-k / 2} \tilde{\sigma}(x) .
\end{aligned}
$$

Then, the Einstein equations (2.4)-(2.8) are linearized in $\tilde{r}, \tilde{\phi}, \tilde{\sigma}$, using

$$
\delta \phi_{, u}=-(-u)^{-k / 2-1}\left(x \tilde{\phi}^{\prime}-\frac{k}{2} \tilde{\phi}\right), \quad \delta \phi_{, v}=-(-u)^{-k / 2+1} \tilde{\phi}^{\prime} .
$$

The resulting equations are homogeneous in $u$, which drops out, and the linearized system reduces to

$$
x \tilde{r}^{\prime \prime}+(-k / 2+3 / 2) \tilde{r}^{\prime}=\frac{\Lambda}{2} e^{2 \sigma}(\tilde{r}+2 \rho \tilde{\sigma}),
$$




$$
\begin{aligned}
& 2 x \tilde{\sigma}^{\prime \prime}+(-k+2) \tilde{\sigma}^{\prime}=\Lambda e^{2 \sigma} \tilde{\sigma}-\left(2 x \psi^{\prime}-1 / 2\right) \tilde{\phi}^{\prime}+(k / 2) \psi^{\prime} \tilde{\phi}, \\
& -(-k+1) x \tilde{r}^{\prime}+\left((-k+1) x \sigma^{\prime}-\left(k^{2}-1\right) / 4\right) \tilde{r}+\rho x \tilde{\sigma}^{\prime}-k\left(x \rho^{\prime}+\rho / 2\right) \tilde{\sigma}= \\
& \quad-\rho\left(x \tilde{\phi}^{\prime}-k\left(1 / 2-x \psi^{\prime}\right) \tilde{\phi}\right)+\left(1 / 4-x \psi^{\prime}\right) \tilde{r}, \\
& 2\left(\rho^{\prime} \tilde{\sigma}^{\prime}+\sigma^{\prime} \tilde{r}^{\prime}\right)-\tilde{r}^{\prime \prime}=\psi^{\prime}\left(2 \rho \tilde{\phi}^{\prime}+\psi^{\prime} \tilde{r}\right), \\
& 2 x \rho \tilde{\phi}^{\prime \prime}+\left(2 x \rho^{\prime}+(-k+5 / 2) \rho\right) \tilde{\phi}^{\prime}-(k / 2) \rho^{\prime} \tilde{\phi}+\left(2 x \psi^{\prime}-1 / 2\right) \tilde{r}^{\prime} \\
& \quad+\left(2 x \psi^{\prime \prime}+(-k / 2+5 / 2) \psi^{\prime}\right) \tilde{r}=0 .
\end{aligned}
$$

What is the number of the independent constants for this system? The perturbed Klein-Gordon equation (5.7) is clearly redundant, while Eqs. (5.5) and (5.6) are constraints. So, as in the $(3+1)$-dimensional case [11, 12], the order of the system is four, and the general solution depends on four integration constants. However, one of these four independent solutions corresponds to a gauge mode and is irrelevant. The parametrisation (4.9) is invariant under infinitesimal coordinate transformations $v \rightarrow v+f(v)$. For $f(v)=-\alpha v^{1+k / 2}$, these lead to $x \rightarrow x-\alpha(-u)^{-k / 2}(-x)^{1+k / 2}$, giving rise to the gauge mode

$$
\begin{aligned}
& \tilde{r}_{k}(x)=\alpha(-x)^{1+k / 2} \rho^{\prime}(x), \\
& \tilde{\phi}_{k}(x)=\alpha(-x)^{1+k / 2} \psi^{\prime}(x), \\
& \tilde{\sigma}_{k}(x)=\alpha\left[(-x)^{1+k / 2} \sigma^{\prime}(x)-\frac{k+2}{4}(-x)^{k / 2}\right],
\end{aligned}
$$

which solves identically the system (5.3)-(5.7). So, up to gauge transformations, the general solution of this system depends only on three independent constants.

These will be determined, together with the possible values of $k$ (the eigenfrequencies) by enforcing appropriate and reasonable boundary conditions. We shall use here the "weak boundary conditions" of [12] on the boundaries $u=0$ and $x=x_{1}(X \rightarrow \infty)$

$$
\lim _{u \rightarrow 0} r^{-1} \neq 0, \quad \lim _{x \rightarrow x_{1}} r \neq 0,
$$

together with the condition

$$
\tilde{r}(0)=0,
$$

which guarantees that the singularity of the perturbed solution starts smoothly from that of the unperturbed one. On the third boundary $v=0$, we shall impose a stronger condition by requiring that the perturbations are analytic in $v$, in order for the perturbed solution to be extendible beyond $v=0$ to negative values of $v$ at finite $u$. 
First, we consider the region $x \rightarrow 0$ where, according to Eqs. (4.1), (4.3), (4.5) and (4.7),

$$
\rho \simeq 1+\frac{1}{3} \Lambda x, \quad e^{2 \sigma} \simeq 1+\frac{4}{15} \Lambda x, \quad \psi \simeq \frac{1}{15} \Lambda x .
$$

Let us assume a power-law behavior

$$
\tilde{r}(x) \sim a(-x)^{p}
$$

where $p$ is a constant to be determined. Then Eqs. (5.3), (5.4) and (5.6) can be approximated near $x=0$ as

$$
\begin{aligned}
& x \tilde{r}^{\prime \prime}+(-k / 2+3 / 2) \tilde{r}^{\prime} \simeq \Lambda \tilde{\sigma} \\
& x \tilde{\sigma}^{\prime \prime}+(-k / 2+1) \tilde{\sigma}^{\prime} \simeq \frac{1}{4} \tilde{\phi}^{\prime} \\
& 2 \rho^{\prime} \tilde{\sigma}^{\prime}-\tilde{r}^{\prime \prime} \simeq 2 \rho \psi^{\prime} \tilde{\phi}^{\prime} .
\end{aligned}
$$

Eliminating the functions $\tilde{\sigma}$ and $\tilde{\phi}$ between these three equations and using Eq. (5.11), we obtain the fourth-order equation

$$
4 x^{2} \tilde{r}^{\prime \prime \prime \prime}+(-4 k+13) x \tilde{r}^{\prime \prime \prime}+(k / 2-1)(2 k-5) \tilde{r}^{\prime \prime} \simeq 0,
$$

which implies the power-law behavior (5.12) with the exponent $p$ constrained by

$$
p(p-1)(p-k / 2-3 / 4)(p-k / 2-1)=0 .
$$

Obviously the root $p=k / 2+1$ corresponds to the gauge mode (5.8) and must be discarded as irrelevant. As a consequence the general solution near $x=0$ can be given in terms of three independent constants as

$$
\begin{aligned}
& \tilde{r}(x) \sim A+B(-x)+\Lambda C(-x)^{3 / 4+k / 2} \\
& \tilde{\sigma}(x) \sim-\frac{A}{2}+\Lambda^{-1} \frac{(k-3) B}{2}-\frac{5 C}{8}\left(k+\frac{3}{2}\right)(-x)^{-1 / 4+k / 2} \\
& \tilde{\phi}(x) \sim \frac{(1-k) A}{2}-\Lambda^{-1} \frac{(k-3) B}{2}+\frac{5 C}{8}\left(k+\frac{3}{2}\right)(-x)^{-1 / 4+k / 2}
\end{aligned}
$$

Let us note that this solution remains valid in the limit $\Lambda \rightarrow 0$, leading to the limiting solution $\tilde{r} \sim A+B(-x)$ (with $B=0$ for $k \neq 3$ ), which could also be obtained directly by solving the equation $\tilde{r}^{\prime \prime}=0$ which results from (5.6) in the limit $\Lambda \rightarrow 0$, together with the stronger condition (from Eq. (5.3)) $(k-3) \tilde{r}^{\prime}=0$. 
Now we enforce the boundary conditions at $x=0$. For $k>0, \tilde{r}$ is dominated by its first constant term in (5.18), so that the condition (5.10) can only be satisfied for $u \rightarrow 0$ if

$$
A=0 \text {. }
$$

Then, for $k>1 / 2, \tilde{r}$ is dominated by its second term $-B x$, leading to a perturbation $(-u)^{1 / 2-k / 2} \tilde{r}(x)$ which blows up as $u \rightarrow 0$ and violates (5.9) unless

$$
k \leq 3 .
$$

Then we impose the condition of analyticity in $v$ at fixed $u$. This is satisfied if

$$
k=2 n-3 / 2,
$$

where $n$ is a positive integer. Combining eqs. (5.22) and (5.23) we find that $k$ has only two positive eigenvalues

$$
k=1 / 2, \quad k=5 / 2 .
$$

However, in the above analysis we have disregarded the fact that $k=1 / 2$ is a double root of the secular equation (5.17). For $k=1 / 2$ the correct behavior of the general solution near $x=0$ is

$$
\begin{aligned}
& \tilde{r}(x) \sim A+B(-x)+\Lambda C(-x) \ln |x|, \\
& \tilde{\sigma}(x) \sim-\frac{A}{2}-\Lambda^{-1} \frac{5 B}{4}-\frac{9 C}{4}-\frac{5 C}{4} \ln |x| \\
& \tilde{\phi}(x) \sim \frac{A}{4}+\Lambda^{-1} \frac{5 B}{4}+\frac{9 C}{4}+\frac{5 C}{4} \ln |x|,
\end{aligned}
$$

which satisfies the condition of analyticity only if $C=0$.

At the AdS boundary $\left(x \rightarrow x_{1}\right)$ the leading behaviour of the background is, from Eqs. (4.24),

$$
\rho \simeq \frac{\rho_{1}}{x-x_{1}}, \quad e^{2 \sigma} \simeq\left(\frac{4 x_{1}}{\Lambda}\right) \frac{1}{\left(x-x_{1}\right)^{2}}, \quad \psi \simeq \psi_{1} .
$$

We again assume a power-law behavior

$$
\tilde{\sigma} \sim b \bar{x}^{q}
$$

$\left(\bar{x}=x-x_{1}\right)$. Then Eq. (5.4), where $\tilde{\phi}$ can be neglected, gives

$$
q(q-1)=2,
$$


i.e. $q=-1$ or $q=2$. Then, Eq. (5.3) reduces near $\bar{x}=0$ to

$$
\tilde{r}^{\prime \prime}-2 \bar{x}^{-2} \tilde{r} \simeq 4 b \rho_{1} \bar{x}^{q-3} .
$$

If $q=-1$, the behavior of the solution is governed by the right-hand side, i.e. $\tilde{r} \propto \bar{x}^{-2}$, which violates the boundary condition (5.9) for $x \rightarrow x_{1}$. So the behavior $\tilde{\sigma} \sim b \bar{x}^{-1}$ must be excluded, which fixes another integration constant $D=0$ (where $D$ is a linear combination of $B$ and $C$ ). Then, the generic behavior of the solution of Eq. (5.31) with $q=2$ is governed by that for the homogeneous equation, i.e.

$$
\tilde{r} \sim \frac{E}{x-x_{1}} .
$$

This is consistent with the boundary condition (5.9), and is an admissible small perturbation if its amplitude is small enough, $E \ll \rho_{1}$.

For $k=1 / 2$, we have seen that two of the three integration constants in (5.25)-(5.27) are fixed $(A=C=0)$ by condition (5.10) and the analyticity condition, while the weak boundary condition at the AdS boundary fixes a third constant $D=0$. However this is impossible, as the perturbation amplitude must remain as a free parameter. So the mode $k=1 / 2$ cannot satisfy all our boundary conditions, and we are left with a single eigenmode,

$$
k=5 / 2,
$$

completely determined up to an arbitrary amplitude by the two conditions $A=D=0$.

The corresponding perturbed metric function $r$ behaves near $x=0$ as

$$
r \simeq(-u)^{1 / 2}\left[1+\frac{1}{3} \Lambda x-(-u)^{-5 / 4} B x\right] .
$$

For $B<0$, the central singularity $r=0$ is approximately given by

$$
(-u)^{1 / 4} \simeq-B v
$$

Our boundary conditions guarantee that it starts at $u=v=0$ (as for the unperturbed solution) and then becomes spacelike in the region $v>0$. This singularity is hidden behind a trapping horizon (defined by Eq. (4.31) which, near $x=0$, is null,

$$
(-u)^{5 / 4}=\frac{3 B}{\Lambda}
$$


(a null trapping horizon was also found in [12]). Let us point out the crucial role played by the cosmological constant $\Lambda$ in the formation of this trapping horizon. For $\Lambda=0, \rho(x)=1$, while, as discussed after Eq. (5.20), the perturbation $\tilde{r}$ with the boundary condition (5.10) vanishes for $\Lambda=0$, so that the perturbed radial function $r$ is (as in [4]) identical to the CSS one, and the trapping horizon does not exist. Near the AdS boundary $x \rightarrow x_{1}$, it follows from (5.28) and (5.32) that both the central singularity and the trapping horizon are tangent to the null line

$$
(-u)^{5 / 4}=-E\left(\frac{4 x_{1}}{\Lambda}\right)^{-1 / 2} .
$$

Thus, perturbations of the quasi-CSS solution lead to black hole formation, showing that this solution is indeed a threshold solution, and is a candidate to describe critical collapse. Near-critical collapse is characterized by a critical exponent $\gamma$, defined by the scaling relation $Q \propto\left|p-p^{*}\right|^{s \gamma}$, for a quantity $Q$ with dimension $s$ depending on a parameter $p$ (with $p=p^{*}$ for the critical solution). Choosing for $Q$ the radius $r_{A H}$ of the apparent horizon, and identifying $p-p^{*}$ with the perturbation amplitude $B$, we obtain from $(5.36)$

$$
r_{A H} \simeq\left(\frac{3 B}{\Lambda}\right)^{2 / 5}
$$

leading to the value of the critical exponent $\gamma=2 / 5$, in agreement with the renormalization group argument [14] leading to $\gamma=1 / k$.

\section{Conclusion}

We have discussed in detail the causal structure of the Garfinkle CSS solutions 2.10 to the $\Lambda=0$ Einstein-scalar field equations. From a special solution of this class, we have derived by a limiting process a new CSS solution, which we have extended to a unique solution of the full $\Lambda<0$ equations, describing collapse of the scalar field onto a null central singularity. This is not a curvature singularity (all the curvature invariants remain finite), but a singularity in the causal structure similar to that of the BTZ black hole. Finally, we have analyzed linear perturbations of the $\Lambda<0$ solution, found a single eigenmode $k=5 / 2$, checked that this mode does indeed give rise to black holes, and determined the critical exponent $\gamma=2 / 5$.

For comparison, Choptuik and Pretorius [2] derived, by analysing the observed scaling behavior of the maximum scalar curvature, the value $1.15<$ $\gamma<1.25$ for the critical exponent. This value is different from the value 
$\gamma \sim 0.81$ obtained in the numerical analysis of Husain and Olivier [3] from the scaling behavior of the apparent horizon radius. Our value $\gamma=0.4$, while significantly smaller than these two conflicting estimates, is of the order of the theoretical value $\gamma=1 / 2$ derived either from the analysis of dust-ring collapse [15], of black hole formation from point particle collisions [16], or of the $J=0$ to $J \neq 0$ transition of the BTZ black hole 17.

It is worth mentioning here that, even though they were obtained for a vanishing cosmological constant and thus solve the $\Lambda \neq 0$ equations only near the singularity, the Garfinkle CSS solutions are, for the particular value (chosen in order to better fit the numerical curves) $c=(7 / 8)^{1 / 2} \simeq 0.935$, in good agreement [国] with the numerical results of [2] at an intermediate time. The fact that this value is close to 1 suggests that the $c=1$ CSS solution (3.3) approximately describes near-critical collapse at intermediate times. If this the case, then it would not be surprising if its late-time limit, our new CSS solution Eq. (3.4), gives a good description of exactly critical collapse near the singularity. A fuller understanding of the relationship between the numerically observed near-critical collapse and these various $\Lambda=0$ CSS solutions could be achieved by extending them to $\Lambda<0$, as done in the present work for the special solution (3.7). 


\section{References}

[1] M. Bañados, C. Teitelboim and J. Zanelli, Phys. Rev. Lett. 69, 1849 (1992); M. Bañados, M. Henneaux, C. Teitelboim and J. Zanelli, Phys. Rev. D 48, 1506 (1993)

[2] F. Pretorius and M.W. Choptuik, Phys. Rev. D 62, 124012 (2000)

[3] V. Husain and M. Olivier, Class. Quant. Grav. 18, L1 (2001)

[4] D. Garfinkle, Pys. Rev. D 63, 044007 (2001)

[5] L. Burko, Phys. Rev. D 62, 127503 (2000)

[6] M. Choptuik, Phys. Rev. Lett. 70, 2980 (1993)

[7] C. Gundlach, Living Rev. Rel. 2, 4 (1999)

[8] M.D. Roberts, Gen. Rel. Grav. 21, 907 (1989)

[9] P.R. Brady, Class. Quantum Grav. 11, 1255 (1994)

[10] Y. Oshiro, K. Nakamura and A. Tomimatsu, Progr. Theor. Phys. 91, 1265 (1994)

[11] A.V. Frolov, Phys. Rev. D 56, 6433 (1997)

[12] S.A. Hayward, Class. Quantum Grav. 17, 4021 (2000)

[13] G. Clément and A. Fabbri, Class. Quantum Grav. 17, 2537 (2000)

[14] T. Koike, T. Hara and S. Adachi, Phys. Rev. Lett. 74, 5170 (1995)

[15] Y. Peleg and A. Steif, Phys. Rev. D 51, 3992 (1995)

[16] D. Birmingham and S. Sen, Phys. Rev. Lett. 84, 1074 (2000) ; D. Birmingham, I. Sachs and S. Sen, "Exact results for the BTZ black hole", hep-th/0102155

[17] J.P. Krisch and E.N. Glass, "Critical exponents for Schwarzschild-Kerr and BTZ systems", gr-qc/0102075 


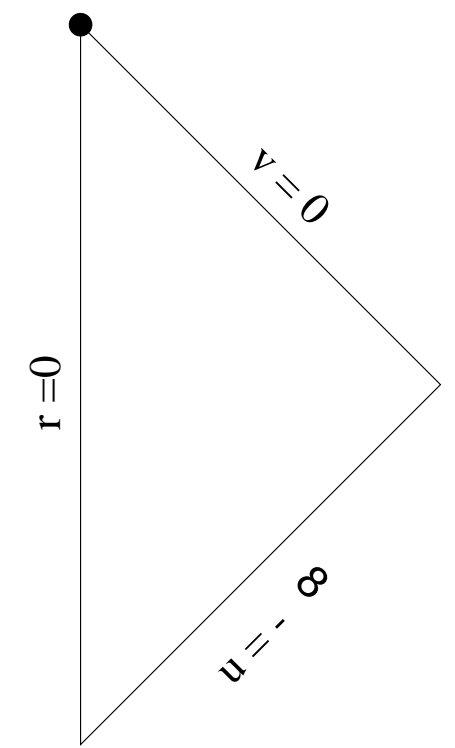

Figure 1: Penrose diagram of the solutions eq. (2.12) for $q<0$.

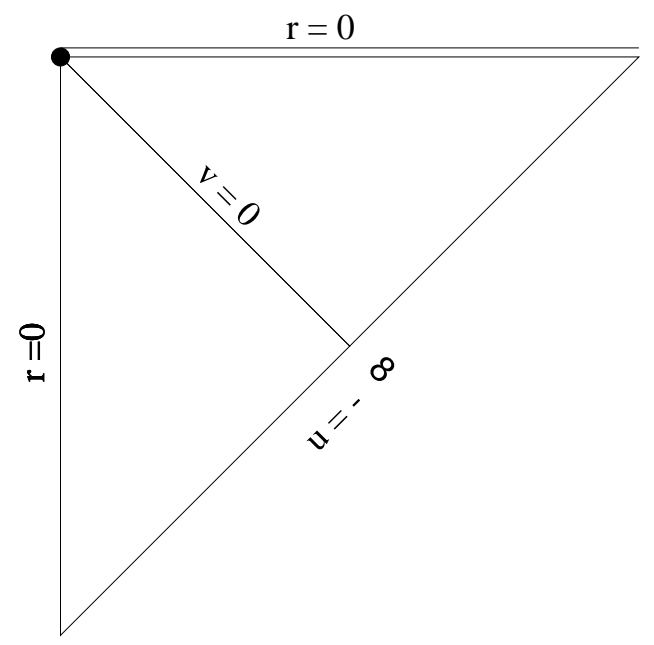

Figure 2: Causal structure for $q=n$ odd. 


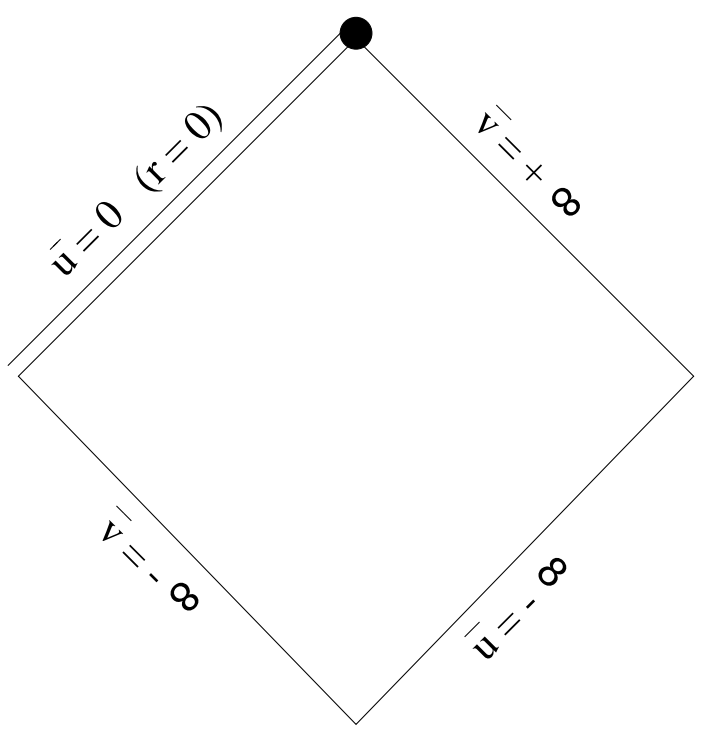

Figure 3: Penrose diagram of our new CSS solution (3.7). The null line $\bar{u}=0$ is a singularity in the causal structure.

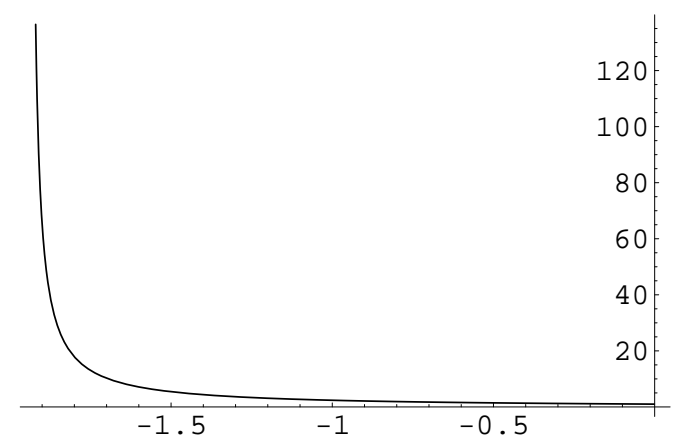

Figure 4: Numerical plot of the function $\rho(x)$ as derived from the system (4.25) with $\rho(0)=0$ and $\rho^{\prime}(0)=-2 / 3$, showing the divergence of $\rho$ for $x \rightarrow x_{1}$ as the AdS boundary is approached (the behaviour is given in the first of Eqs. (4.24). 


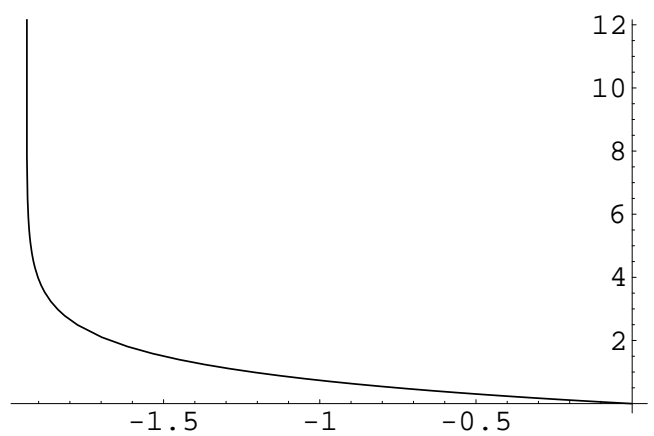

Figure 5: Numerical graph of $\sigma(x)$ starting from $\sigma(0)=0$. In the limit $x \rightarrow x_{1}$ this is well represented in the second of Eqs. (4.24).

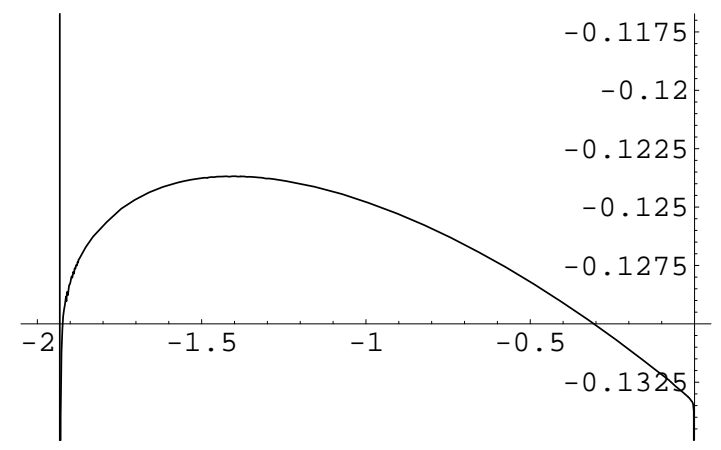

Figure 6: Plot of $\psi^{\prime}(x)$. In particular it is clear that $\psi^{\prime \prime}(x) \rightarrow \infty$ as $x \rightarrow x_{1}$. This feature is reproduced in the third of Eqs. 4.24) (giving $\psi^{\prime \prime} \sim \ln (x-$ $\left.x_{1}\right)$ ). 